NOTICE: this is the author's version of a work that was accepted for publication in Complementary Therapies in Medicine. Changes resulting from the publishing process, such as peer review, editing, corrections, structural formatting, and other quality control mechanisms may not be reflected in this document. Changes may have been made to this work since it was submitted for publication. A definitive version was subsequently published in Bishop FL, Zaman S, Lewith GT (2011). Acupuncture for low back pain: a survey of clinical practice in the UK. Complementary Therapies in Medicine 19, 144148. http://dx.doi.org/10.1016/j.ctim.2011.03.004

\title{
Acupuncture for low back pain: a survey of clinical practice in the UK
}

Authors: Felicity L Bishop PhD, Shipu Zaman, George T Lewith DM.

Institutional Affiliation: University of Southampton School of Medicine, Southampton General Hospital, Tremona Road, Southampton, SO16 6YD, UK.

\section{Corresponding author:}

Dr Felicity Bishop. Primary Medical Care, Aldermoor Health Centre, Aldermoor Close, Southampton, UK, SO16 5ST. Email: F.L.Bishop@southampton.ac.uk. Phone: +44(0)23 8024 1072. Fax: +44(0)23 80701125 .

\section{Sources of Financial Support:}

Dr Bishop is funded by a non-clinical career development Fellowship from Arthritis Research UK (grant number 18099). Mr Zaman is a student at the University of Southampton School of Medicine; he carried out this survey as part of his Study in Depth. Professor Lewith is partially supported by a grant from the Rufford Maurice Laing Foundation. The funders and sponsors had no role in the study design, in the collection, analysis and interpretation of data; in the writing of the manuscript; or in the decision to submit the manuscript for publication

Main Text Word Count: 2714 


\begin{abstract}
Objectives

Acupuncture is recommended in official UK guidelines for persistent non-specific low back pain and is popular with patients. However, what UK-based acupuncturists actually do in every day clinical practice is poorly documented. We therefore conducted a survey of every-day clinical practice of acupuncture for low back pain in the UK.
\end{abstract}

\title{
Design
}

Cross-sectional postal survey.

\section{Setting}

Random samples of 100 acupuncturists from each of the three main UK societies for acupuncturists were sent questionnaires.

\section{Main Outcome Measures}

A questionnaire designed (and pilot-tested) for this study asked about: training and professional identity, current work situation, and typical approach to treating a patient with low back pain.

\section{$\underline{\text { Results }}$}

Completed questionnaires were received from 129 respondents ( $53 \%$ male), representing each society approximately equally. Work situation (e.g. weekly hours practicing acupuncture) differed across societies. Respondents reported needling 2 to 30 acupuncture points in an average treatment (median $=8$ ) and leaving needles in for 0 to 40 minutes (median $=20$ minutes). A large number of individual points (121) were named as 'typical'.

\section{Conclusions}

There is huge variation in how acupuncture is used by UK practitioners to treat people with low back pain. This probably constitutes a difficult situation for patients when selecting an acupuncturist. It may also translate into large variation in clinical outcomes for patients receiving care from different acupuncturists as well as difficulty in developing acupuncture protocols for pragmatic and other trials. .Such diversity reflects the ongoing debate within the acupuncture profession about good practice for common conditions. 


\section{Introduction}

Acupuncture has recently been included in official UK guidelines for persistent nonspecific low back pain ${ }^{1}$. Indeed, recent systematic reviews suggest acupuncture can be effective (without necessarily having specific efficacy) for non-specific low back pain ${ }^{2 ; 3}$. However, comparatively little is known about how acupuncture is actually used in every day clinical practice. The bulk of the published literature on acupuncture for low back pain consists of case reports, case series, or clinical trials ${ }^{4}$. The every-day clinical practice of acupuncture has received less attention, although Yuan and colleagues ${ }^{5}$ found important and potentially clinically significant differences in treatment regimens for low back pain when they compared regimens described in published RCTs, textbooks, expert opinion pieces, surveys and case studies. While some trials have made considerable efforts to create a treatment protocol that reflects normal clinical practice in the terms of point prescription, frequency and duration of treatment ${ }^{6 ; 7}$ this has certainly not been the case across the board for all acupuncture studies. This may be a reflection of the diverse opinions and training schools throughout the UK with some courses adopting a very Western approach while others utilise a variety of traditional methods. ${ }^{8}$

It is important to know how acupuncture is used in clinical practice so that we can inform future pragmatic trials and work towards defining best practice. Physician and non-physician acupuncturists in the United States have been surveyed ${ }^{9 ; 10}$, treatment records of US-based acupuncturists have been described ${ }^{11}$, and international acupuncture experts have been surveyed ${ }^{12}$. However, there are many and sometimes conflicting approaches to acupuncture in the West and thus great potential for variety in practice. White ${ }^{13}$ describes Western medical acupuncture as a distinctly different entity to a Traditional Chinese Medicine (TCM) approach to diagnosis and treatment which in turn may differ from a Five Element approach to diagnosis and therapy. Within a Western approach the main aim in terms of both diagnosis and treatment is its focus on pain and musculoskeletal conditions such as osteoarthritis. A Western acupuncturist will concentrate on treating the local tender points (often classical acupuncture points) that appear to act by stimulating the nervous system through known modes of action. A TCM acupuncturist may choose to treat local tender points but will also usually include some non tender acupuncture points based on a TCM diagnosis. This takes into account the bodies 'energies' by using a combination of history and tongue and pulse diagnosis which claims to assess the individual's balance of Yin and Yang and the circulation of Qi. 
In the UK, there is currently no statutory regulation of acupuncture, although professions such as registered medical practitioners and physiotherapists incorporating acupuncture into their practice are subject to statutory regulation. Acupuncturists belong to one (or more) of three major organisations, the Acupuncture Association of Chartered Physiotherapists (AACP, for physiotherapists), the British Acupuncture Council (BAcC), and the British Medical Acupuncture Society (BMAS, for medically qualified practitioners). It is likely that the BMAS will contain a majority of Western acupuncturists but all three organisations will have people practising very different types of acupuncture within their professional bodies. What these acupuncturists actually do in both the NHS and private every day clinical practice is not well documented. We therefore decided to conduct a survey of every-day clinical practice of acupuncture for low back pain in the UK.

\section{Methods}

\section{Design}

This was a cross-sectional self-report postal questionnaire survey.

\section{Materials}

A short questionnaire (31 items) was designed for this study (Appendix). Questions asked about: training and professional identity (or identities); current work situation including number of hours of acupuncture per week; typical approach to treating a patient with low back pain, including point selection and co-interventions. Pre-testing was achieved by seeking comments on the questionnaire from five acupuncturist members of the researchers' group. This ensured the questionnaire was understandable and had acceptable face validity.

\section{Procedure}

A random sample of 100 acupuncturists was obtained (using a random number generator) from listings of each of the three professional societies (AACP, BMAS, $B A c C$ ). Each selected acupuncturist was sent, by post, a single copy of the questionnaire accompanied by a covering letter and a freepost reply envelope. Questionnaires were completed anonymously and returned directly to the researchers. Ethical approval was obtained from the host institution. 


\section{Analytic Methods}

Simple descriptive statistics (mean, standard deviation, median) were used to describe the respondents and their practices. Pearson's chi-squared and Kruskal-Wallis tests were used to evaluate differences between members of the three societies.

\section{Results}

\section{Respondents}

Completed questionnaires were received from 129 respondents (response rate $=43 \%$ ). There were no means to establish the eligibility of those who did not respond (e.g. some may have retired) or to examine any differences between responders and nonresponders.

Respondents were aged between 25 and 72 years (median $=51$ ) and slightly over half were male $(68,53 \%)$. Approximately even numbers of respondents were registered with the BMAS $(48,37 \%)$ and the AACP $(45,35 \%)$, with slightly fewer registered with the $\mathrm{BAcC}(36,28 \%)$. The majority of respondents were also registered practitioners of other disciplines: $35 \%(n=45)$ were doctors, $35 \%(n=45)$ were physiotherapists, smaller numbers (4 or less) were osteopaths, herbalists, homeopaths, and nurses. This differed across the societies. Almost all BMAS members (43, 92\%) were doctors, almost all AACP members $(44,98 \%)$ were physiotherapists, and BAcC members reporting a variety of other professions: 4 herbalists $(11 \%), 3$ nurses $(8 \%), 2$ osteopaths (6\%), 1 doctor, 1 physiotherapist, and 1 homeopath ( $3 \%$ each). Most respondents had undertaken part-time training in acupuncture (94,74\%), and the duration of initial training ranged from 30 hours to 7 years (median $=9$ months). This differed across the societies (Pearson's $\left.X^{2}(2, N=123)=64.6, p<.01\right)$ : All BAcC members $(n=36)$ had 12 months or more initial acupuncture training, $91 \%(n=41)$ of AACP members had less than 12 months, $52 \%(n=25)$ of BMAS members had less than 12 months.

Just over half of the respondents worked full time $(74,57 \%)$. The average time spent practicing acupuncture each week varied greatly from 0 to 45 hours (median $=8$ ), and was significantly different across members of the three societies (Kruskall-Wallis $x^{2}$ $(2, N=124)=37.5, p<.01)$. The median number of hours practicing each week was highest among BAcC members (18 hours), followed by BMAS ( 6 hours) and then AACP (3 hours). Respondents reported having practiced acupuncture for between 8 and 368 months $($ median $=120$, i.e. 10 years). 
Overall, a small proportion of respondents worked within the public sector (18, $14 \%)$, the majority worked in private practice $(74,57 \%)$, and the remainder worked in both the public and private sectors $(37,29 \%)$. This pattern differed significantly across the societies (Pearson's $\left.X^{2}(4, N=128)=45.9, p<.01\right)$. BMAS practitioners mainly worked in the private sector $(22,47 \%)$ or in both sectors $(23,49 \%)$, while BAcC members almost exclusively worked in the private sector $(33,97 \%)$. AACP members were more evenly split between the NHS $(16,36 \%)$, the private sector $(18,40 \%)$, and both $(11,25 \%)$.

\section{Treating Patients with Back Pain}

Respondents reported seeing, on average, between 1 and 40 new patients each month with back pain (median $=6)$. This differed across societies (Kruskall-Wallis $\mathrm{X}^{2}(2 \mathrm{~N}=122)$ $=24.0, p<.01)$. AACP members saw more new patients with back pain (median $=10$ ) than BMAS members (median $=5$ ) who in turn saw more than BAcC members (median = 3.5). Slightly more respondents used Western acupuncture $(52,41 \%)$ than used TCM acupuncture $(30,23 \%)$, and many used a mixture of the two $(46,36 \%)$ and again this differed across organisations (Pearson's $\left.X^{2}(4, N=127)=97.7, p<.01\right)$. As Figure 1 shows, BAcC members predominantly used TCM acupuncture while AACP and BMAS members used Western acupuncture alone or in combination with TCM.

Most participants $(84,65 \%)$ would initially treat someone with low back pain on a weekly basis while some $(35,27 \%)$ would see them twice a week. A large majority of respondents $(104,81 \%)$ reported changing the frequency of treatments over time. Decisions about treatment frequency were often based on more than one consideration, but many acupuncturists reported being primarily influenced by patient improvement (for $50,39 \%$ of acupuncturists) or pain levels and the impact of pain on the patient's activities $(31,24 \%)$. The patient's availability and the practitioner's availability were also taken into account but were less frequently cited as primary determinants of treatment frequency.

Respondents reported needling between 2 and 30 acupuncture points in an average treatment (median $=8$ ) and leaving needles in for between 0 and 40 minutes (median $=20$ minutes). These factors were not associated with duration of initial training in acupuncture or with acupuncture style (Western/TCM/mixed). Needling time did differ across societies (Kruskall-Wallis $\left.X^{2}(2 \mathrm{~N}=124)=29.7, p<.01\right)$ and BMAS members left needles in for less time (median $=14$ minutes) than BAcC members (median $=20$ minutes) or AACP members (median $=20$ minutes). There was also a trend for BMAS 
members to needle more acupuncture points in an average session (median for BMAS = 9, for $\mathrm{AACP}=8$, for $\mathrm{BAcC}=8$; Kruskall-Wallis $\left.\mathrm{x}^{2}(2 \mathrm{~N}=121)=5.6, \mathrm{p}=.06\right)$.

Most commonly, respondents aimed to elicit needling sensation on almost all occasions $(46,36 \%)$ or most of the time $(43,33 \%)$. Fewer aimed to elicit needling sensation frequently $(24,19 \%)$, occasionally $(10,8 \%)$ or not at all $(5,4 \%)$. We asked respondents to list those acupuncture points that they commonly select during treatment of patients with lower back pain. A large number of points (121) were listed. Points named by more than a quarter of the sample (32 respondents), were: Bladder Meridian (BL) 23, BL25, BL60, BL40, BL26, BL54, and Gallbladder Meridian (GB) 30. More generally, 45 respondents (35\%) reported using trigger points. Additional points named by between 10 and 32 participants (8-25\%) were: Governor Vessel (GV) 4, BL24, GB34, B28, Kidney Meridian (KI)3, BL62, GV3, Large Intestine Meridian (LI) 4, BL27, Liver Meridian (LR) 3, BL32, BL57, BL36, BL22, Small Intestine Meridian (SI) 3. Points named by fewer than 10 respondents were on the following meridians: Conception Vessel, GV, GB KI, Stomach, Spleen, Triple Energiser, BL, LI, LR, and SI.

We asked respondents to rate, on a scale of 1 (not at all) to 5 (very much), the extent to which different factors influence their point selection when treating people with low back pain. The most strongly endorsed factor was experience $(M=4.2, S D=.9)$, followed by selecting points at the exact location of pain (tender points) $(\mathrm{M}=3.5, \mathrm{SD}=$ $1.1)$, and selecting distal points on the meridian running over the pain $(M=3.3, S D=$ 1.3). Respondents also considered their specific point selection to be influenced by their training $(M=2.6, S D=1.2)$ and TCM diagnosis $(M=2.4, S D=1.5)$, but these factors were rated as slightly less influential on average.

Table 1 shows the extent to which respondents used other interventions in addition to acupuncture when treating people with low back pain. The most commonly used co-interventions were exercise (used by almost all respondents) and massage, while herbal remedies and cupping were used by a minority of respondents. Moxibustion was used by $36 \%$ of respondents.

\section{Discussion}

There is huge variation in how acupuncture is used to treat people with low back pain in the UK. Our respondents were experienced practitioners who used acupuncture in a wide variety of ways and settings to treat people with back pain in every day clinical practice. Practitioners based their point selection primarily on clinical experience but 
also on their initial training, selecting points based on the location of pain and distal points on the meridian running over the pain. Some of the variability in practice was traced back to the societies through which our respondents were recruited, and which themselves differ in the training and qualifications they provide for and require of their members. Members of the BAcC worked in the private sector and practiced acupuncture for much longer each week than members of the BMAS or the AACP, but members of the AACP (who worked across the NHS and private practice) saw more patients with back pain. People with low back pain tend to be seen by acupuncture practitioners who are also physiotherapists and may thus be more likely to receive western acupuncture or a combination of western and TCM acupuncture.

Our survey was based on a random sample of acupuncturists from all three main acupuncture societies in the UK. However, our response rate was somewhat disappointing $(43 \%)$ and we have been unable to test the extent to which our responders constitute a biased sample. The questionnaire items used to assess training were somewhat limited, and future surveys should obtain more precise details for example about qualifications obtained and training providers.

There are some similarities between our findings and other studies. Our respondents used various other therapies alongside acupuncture, including both conventional and complementary/alternative therapies; acupuncturists in the York Acupuncture for Back Pain trial similarly used a range of auxiliary treatments ${ }^{14}$. The considerable variation that we found (in the number of points used in an average treatment, the duration of needling and the specific points chosen) echoes that found among US acupuncturists ${ }^{10}$. There was some overlap between the points named by our respondents and those reported in other surveys, for example at least 10 of our respondents named each of BL23, BL40, GB34, BL54, and BL60, which were the points preferred by acupuncture experts ${ }^{12}$. There was also substantial overlap with points used in published clinical trials. For example, BL23, BL25, and BG30 were mentioned by most of our respondents and were standard points in the trial by Molsberger and colleagues ${ }^{15}$. The German Acupuncture Randomised Trial in low back pain ${ }^{16}$ allowed acupuncturists to choose points from BL20 to BL34, BL50 to BL54, GB30 and GV3-6, many of which were mentioned by our respondents. In the GERAC low back pain trial the obligatory points for dorsal pain were $B L 23,40,60$, and $K I 3$, and for lateral pain were $\mathrm{BL} 23, \mathrm{~GB} 34,41$, and $\mathrm{KI}^{7}{ }^{7}$, all of which (except GB41) were mentioned by more than 10 of our respondents. 
The findings have implications for trial design, patients, and policy. The variety of practices described by respondents (e.g. naming 121 individual points as typical) suggests that to produce a truly pragmatic trial that reflects clinical practice triallists need to give acupuncturists free choice of both co-interventions and acupuncture points and styles ${ }^{17 ; 18}$. For patients, differences between acupuncturists may be difficult to identify in advance and thus could prove challenging when seeking an individual acupuncturist. Those who want traditional Chinese acupuncture or a practitioner who has undertaken at least one year of initial acupuncture training would be more likely to find these qualities in a BAcC member; such details could be made more accessible to patients seeking care. The variety in practices might well translate into variations in effectiveness and patients' experiences, but this goes beyond the scope of a survey and is difficult to establish based on published evidence. For example: while some points used by our respondents have been used in clinical trials others have not; while many respondents aimed to elicit needling sensation at present we do not fully understand how needling sensation relates to treatment outcome; six or more treatments may be more effective than fewer treatments ${ }^{19}$,but whether the frequency of initial treatments (weekly or biweekly) affects outcomes is not clear. For policy, the variety in practices will make it very difficult to establish best practice guidelines for the provision of acupuncture within the NHS. One consequence of statutory regulation and associated changes in acupuncture education (should they occur in the UK as seems probable) might be to decrease the variability in acupuncture practices ${ }^{8}$.

Our survey should be replicated and extended to a larger potential pool of respondents to ensure the generalisability of our findings. However, doing so would be unlikely to change our primary conclusion that there is huge variety in how acupuncture is used by UK practitioners to treat people with low back pain. Future work should explore the reasons for this diversity and investigate in more detail the various factors that influence established clinical practice. Large scale surveys and small scale qualitative work could contribute to this endeavour. We also need to understand the comparative effectiveness of different approaches to using acupuncture for low back pain. 


\section{Acknowledgements}

We would like to thank all those who participated in our study by filling in a questionnaire. 
Table 1. Frequency of use of other interventions to treat people with low back pain in addition to acupuncture

\begin{tabular}{llll}
\hline Co-Intervention & Used not at all & $\begin{array}{l}\text { Used Occasionally } \\
\text { or Frequently }\end{array}$ & $\begin{array}{l}\text { Used most or all of } \\
\text { the time }\end{array}$ \\
\hline Herbal remedies & $106(82 \%)$ & $19(15 \%)$ & 0 \\
Exercise & $8(6 \%)$ & $49(38 \%)$ & $69(54 \%)$ \\
Conventional drugs & $54(42 \%)$ & $46(35 \%)$ & $22(17 \%)$ \\
Massage & $43(33 \%)$ & $64(50 \%)$ & $19(15 \%)$ \\
Manipulation & $68(53 \%)$ & $45(35 \%)$ & $12(9 \%)$ \\
Moxibustion & $82(64 \%)$ & $32(24 \%)$ & $12(9 \%)$ \\
Cupping & $92(71 \%)$ & $29(23 \%)$ & $5(4 \%)$ \\
Electro-acupuncture & $45(35 \%)$ & $64(50 \%)$ & $17(13 \%)$ \\
Diet & $53(41 \%)$ & $65(51 \%)$ & $6(6 \%)$ \\
\hline
\end{tabular}

Note $-\%$ is given out of total responses to each item. 


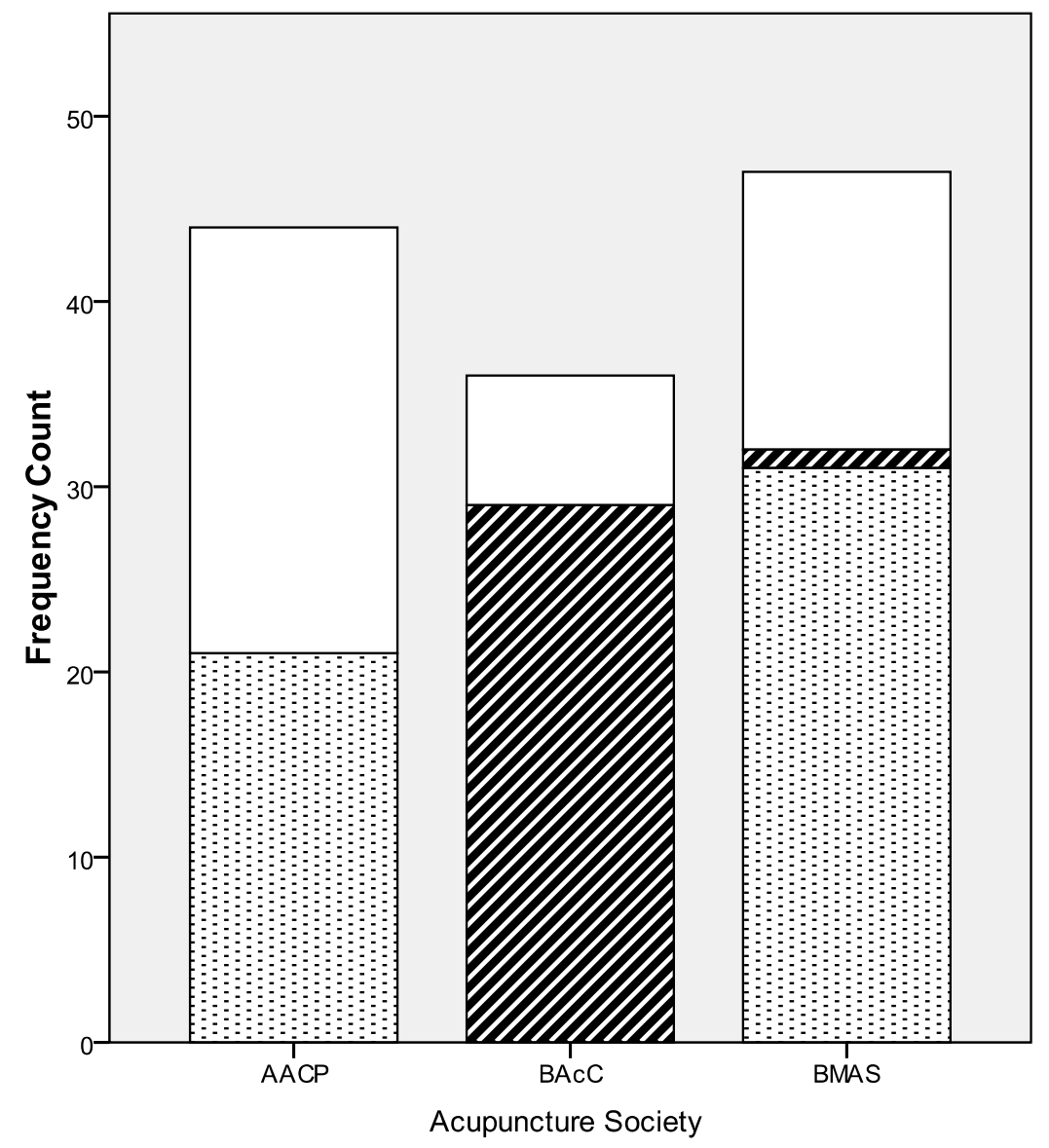

Acupuncture Style

$\square$ Mixed

TTCM

GWestern

Figure 1. Acupuncture style used by members of each society. 
Reference List

(1) Savigny P, Kuntze S, Watson P, Underwood M, Ritchie G, Cotterell M et al. Low back pain. Early management of persistent non-specific low back pain. NICE clinical guideline 88, www.nice.org.uk/CG88. 2009. London, National Collaborating Centre for Primary Care and Royal College of General Practitioners.

(2) Yuan JP, Purepong NM, Kerr DPP, Park JK, Bradbury IP, McDonough SP. Effectiveness of Acupuncture for Low Back Pain: A Systematic Review. [Review]. Spine 2008; 33(23):E887-E900.

(3) Manheimer E, White A, Berman B, Forys K, Ernst E. Meta-analysis: acupuncture for low back pain. Ann Intern Med 2005; 142:651-663.

(4) Lewis KM, Abdi SM. Acupuncture for Lower Back Pain: A Review. [Review]. Clinical Journal of Pain 2010; 26(1):60-69.

(5) Yuan J, Kerr D, Park J, Liu XH, McDonough S. Treatment regimens of acupuncture for low back pain--A systematic review. Complement Ther Med 2008; 16(5):295-304.

(6) Haake M, Muller H-H, Schade-Brittinger C, Basler HD, Schafer H, Maier C et al. German acupuncture trials (GERAC) for chronic low back pain. Randomized, multicenter, blinded, parallel-group trial with 3 groups. Arch Intern Med 2007; 167(17):1892-1898.

(7) Haake M, Muller HH, Schade-Brittinger C, Prinz H, Basler HD, Streitberger K et al. The German Multicenter, Randomized, Partially Blinded, Chronic Low-Back Pain: A Preliminary Report on the Prospective Trial of Acupuncture for Rationale and Design of the Trial. The Journal of Alternative and Complementary Medicine 2003; 9(5):763-770.

(8) Birch SJ, Felt RL. Understanding Acupuncture. London: Churchill Livingstone; 1999.

(9) Kalauokalani DM, Cherkin DCP, Sherman KJP. A Comparison of Physician and Nonphysician Acupuncture Treatment For Chronic Low Back Pain. [Article]. 
Clinical Journal of Pain Childhood Abuse and Pain in Adulthood 2005; 21(5):406-411.

(10) Sherman KJ, Hogeboom CJ, Cherkin DC. How traditional Chinese medicine acupuncturists would diagnose and treat chronic low back pain: results of a survey of licensed acupuncturists in Washington State. Complement Ther Med 2001; 9(3):146-153.

(11) Sherman KJ, Cherkin DC, Hogeboom CJ. The Diagnosis and Treatment of Patients with Chronic Low-Back Pain by Traditional Chinese Medical Acupuncturists. The Journal of Alternative and Complementary Medicine 2001; 7(6):641-650.

(12) Molsberger AF, Zhou J, Arndt D, Teske W. Chinese Acupuncture for Chronic Low Back Pain: An International Expert Survey. The Journal of Alternative and Complementary Medicine 2008; 14(9):1089-1095.

(13) White A, the editorial board of Acupuncture in Medicine. Western medical acupuncture: a definition. Acupuncture in Medicine 2009; 27(1):33-35.

(14) MacPherson H, Thorpe L, Thomas K, Campbell M. Acupuncture for low back pain: traditional diagnosis and treatment of 148 patients in a clinical trial. Complement Ther Med 2004; 12(1):38-44.

(15) Molsberger AF, Mau J, Pawelec DB, Winkler J. Does acupuncture improve the orthopedic management of chronic low back pain - a randomized, blinded, controlled trial with 3 months follow up. Pain 2002; 99(3):579-587.

(16) Brinkhaus B, Witt CM, Jena S, Linde K, Streng A, Wagenpfeil S et al. Acupuncture in patients with chronic low back pain - A randomized controlled trial. Arch Intern Med 2006; 166(4):450-457.

(17) Thomas KJ, MacPherson H, Thorpe L, Brazier J, Fitter M, Campbell MJ et al. Randomised controlled trial of a short course of traditional acupuncture compared with usual care for persistent non-specific low back pain. BMJ 2006; 333(7569):623.

(18) Witt CM, Jena S, Selim D, Brinkhaus B, Reinhold T, Wruck K et al. Pragmatic randomized trial evaluating the clinical and economic effectiveness of 
acupuncture for chronic low back pain. American Journal of Epidemiology 2006; 164(5):487-496.

(19) Ezzo J, Berman B, Hadhazy VA, Jadad AR, Lao L, Singh BB. Is acupuncture effective for the treatment of chronic pain? A systematic review. Pain 2000; 86(3):217-225. 